\title{
OSCILLATION AND NONOSCILLATION OF EVEN-ORDER NONLINEAR DELAY-DIFFERENTIAL EQUATIONS*
}

\author{
$\mathrm{BY}$ \\ BHAGAT SINGH (University of Wisconsin Center, Manitowoc)
}

1. Abstract. The purpose of this paper is to compare the equations

$$
\begin{aligned}
& y^{(2 n)}(x)+p(x) g\left(y(x), y_{\tau}(x)\right)=0 \\
& y^{(2 n)}(x)+p(x) g\left(y(x), y_{\tau}(x)\right)=f(x)
\end{aligned}
$$

for their oscillatory and nonoscillatory nature. In Eqs. (1) and (2) $y^{(i)}(x) \equiv$ $\left(d^{i} / d x^{i}\right) y(x), i=1,2, \cdots, 2 n ; y_{\tau}(x) \equiv y(x-\tau(x)) ; d y / d x$ and $d^{2} y / d x^{2}$ will also be denoted by $y^{\prime}$ and $y^{\prime \prime}$ respectively. Throughout this paper it will be assumed that $p(x)$, $f(x), \tau(x)$ are continuous real-valued functions on the real line $(-\infty, \infty) ; f(x), p(x)$ and $\tau(x)$, in addition, are nonnegative, $\tau(x)$ is bounded and $f(x), p(x)$ eventually become positive to the right of the origin. In regard to the function $g$ we assume the following:

(i) $g: R^{2} \rightarrow R$ is continuous, $R$ being the real line,

(ii) $g(\lambda x, \lambda y)=\lambda^{2 a+1} g(x, y)$ for all real $\lambda \neq 0$ and some integer $q \geq 1$,

(iii) $\operatorname{sgn} g(x, y)=\operatorname{sgn} x$,

(iv) $g(x, y) \rightarrow \infty$ as $x, y \rightarrow \infty ; g$ is increasing in both arguments monotonically.

Eq. (1) is called oscillatory if every nontrivial solution $y(t) \in\left[t_{0}, \infty\right)$ has arbitrarily large zeros; i.e., for every such solution $y(t)$, if $y\left(t_{1}\right)=0$ then there exists $t_{2}>t_{1}$ such that $y\left(t_{2}\right)=0$. Eq. (1) is called nonoscillatory if it has a solution with a last zero or no zero in $\left[t_{0}, \infty\right), t_{0} \geq a>0$. A similar definition holds for eq. (2). All solutions of (1) and (2) considered henceforth are continuous and nontrivial, existing on some halfline $\left[t_{0}, \infty\right)$.

2. Nonoscillation of Eq. (1). We will need the following lemmas.

Lemma 1. (Staikos and Petsoulas [8, p. 697].) If $y(t)>0, y^{\prime}(t)>0$ and $y^{\prime \prime}(t) \leq 0$ for large $t$, then $\lim _{t \rightarrow \infty}\left(y_{r}(t) / y(t)\right)=1$.

Lemma 2. Suppose $\int^{\infty} t^{2 n-1} p(t) d t=\infty$. Let $y(t)$ be a nonoscillatory solution of Eq. (2) such that $y(t)<0$ for large $t$. Then

$$
y(t)<0, y^{\prime}(t)<0,(-1)^{i} y^{(i)}(t) \geq 0, \quad i=2,3, \cdots 2 n .
$$

Proof. Let $T$ be large enough so that $y(t)$ and $y_{\tau}(t)$ are both negative for $t \in[T, \infty)$. Since $f(t) \geq 0$ for large $t$, it follows from Eq. (2) that

$$
y^{(2 n)}(t) \geq f(t) .
$$

This implies that $y^{(2 n-1)}(t) \leq 0$ eventually. In fact, if $y^{(2 n-1)}(t)>0$ eventually then $y^{(2 n-1)}>0$ and $y^{(2 n)}(t) \geq 0$ imply that $y^{(2 n-2)}(t)$ is concave up and increasing. Thus

* Received May 4, 1972; revised version received August 4, 1972. The author is grateful to the referee for his valuable suggestions for improvements in this manuscript. 
$y^{(2 n-2)}(t)>0$ eventually. Similarly $y^{(2 n-3)}(t)>0$ eventually. Proceeding this way, we find that $y(t)>0$ eventually, a contradiction. In a similar manner we find that $y^{\prime}(t)<0$ eventually. For the rest of the conclusion of the lemma, we only need to consider when $y(t) \rightarrow-\infty$, since the conclusion is true if $\lim _{t \rightarrow \infty} y(t)>-\infty$. We shall show that $y^{(2 n-2)}(t) \geq 0$ eventually. Suppose to the contrary that $y^{(2 n-2)}(t)<0$ eventually. Let $T_{1}>T$ so that for $t \geq T_{1}, y^{(2 n-2)}(t)<0$. Integrating (2) between $\left[T_{1}, t\right]$, we have

$$
\begin{aligned}
y^{(2 n-1)}(t) & =y^{(2 n-1)}\left(T_{1}\right)-\int_{T_{1}}^{t} p(s) g\left(y(s), y_{\tau}(s)\right) d s+\int_{T_{1}}^{t} f(s) d s \\
& \geq y^{(2 n-1)}\left(T_{1}\right)-\int_{T_{1}}^{t} p(s) g\left(y(s), y_{\tau}(s)\right) d s,
\end{aligned}
$$

whence

$$
\lim _{t \rightarrow \infty} \int_{T_{1}}^{t} p(s) g\left(y(s), y_{\tau}(s)\right) d s>-\infty,
$$

since $y^{(2 n-1)}(t) \leq 0$ for $t \geq T_{1}$. Since $y^{(2 n-2)}(y)<0$ and $y^{(2 n-1)}(t) \leq 0$, there exists a constant $R_{0}>0$ such that $-y^{(2 n-2)} \geq R_{0}$, which yields, on repeated integration, that

$$
\lim _{t \rightarrow \infty}\left(-y / t^{2 n-2}\right) \geq R>0 \text {. }
$$

From (5), we get

$$
\lim _{t \rightarrow \infty} \int_{T_{1}}^{t} s^{2 n-1} \frac{p(s) g\left(-y(s),-y_{\tau}(s)\right)}{s^{2 n-1}} d s<\infty
$$

and since

$$
\lim _{t \rightarrow \infty} \int_{T_{1}}^{l} s^{2 n-1} p(s) d s=\infty
$$

we must have

$$
\lim _{t \rightarrow \infty} \frac{g\left(-y(s),-y_{\tau}(s)\right)}{s^{2 n-1}}=0
$$

Now if $0 \leq \tau(t) \leq m$,

$$
\begin{aligned}
\frac{g\left(-y(s),-y_{\tau}(s)\right)}{s^{2 n-1}} & \geq \frac{g\left(-y_{\tau}(s),-y_{\tau}(s)\right)}{s^{2 n-1}} \\
& \geq \frac{g(-y(s-m),-y(s-m))}{s^{2 n-1}} \\
& =\frac{[-y(s-m)]^{2 a+1} g(1,1)}{s^{2 n-1}} \\
& \rightarrow 0 \text { as } s \rightarrow \infty .
\end{aligned}
$$

Therefore

$$
\lim _{x \rightarrow \infty} \frac{-y^{2 a \cdot 1}(s-m)}{\left.s^{2 n-1}-m\right)}=\lim _{x \rightarrow \infty} \frac{y^{2 u}}{s} \cdot \lim _{x \rightarrow \infty} \frac{(-y)}{s^{2 n-2}} \geq R \lim _{s \rightarrow \infty} \frac{y^{2 n}}{s}
$$


and, because of (6), we have

$$
\lim _{s \rightarrow \infty} \frac{y^{2 a}(s-m)}{s}=0 .
$$

Since $q \geq 1$ and $\lim _{t \rightarrow \infty} y^{2 q-1}(t-m)=-\infty$, we have

$$
\lim _{t \rightarrow \infty} \frac{y(t-m)}{t}=0 \Rightarrow y^{\prime}(t-m) \rightarrow 0 \quad \text { as } \quad t \rightarrow \infty \text {. }
$$

Since all subsequent derivatives up to order $(2 n-1)$ are monotonic we must have $y^{(2 n-2)}(t) \rightarrow 0$ as $t \rightarrow \infty$, a contradiction. Hence $y^{(2 n-2)}(t) \geq 0$ eventually. The rest of the conclusion can be proved similarly. The proof is complete.

Theorem 1. If $\int^{\infty} t^{2 n-1} p(t) d t=\infty$, then all nonoscillatory solutions of Eq. (2) are nonnegative.

Proof. Suppose, to the contrary, that a nonoscillatory solution $y(t)$ of (2) is eventually negative. Let $T_{1}$ be large enough so that $y(t), y_{\tau}(t)$ are negative for $t \geq T_{1}$.

From Lemma (2), there exists $T_{2} \geq T_{1}$ such that for $t \geq T_{2}$

$y(t)<0, y^{\prime}(t)<0, y^{\prime \prime}(t) \geq 0, y^{\prime \prime \prime}(t) \leq 0, \cdots, y^{(2 n-2)}(t) \geq 0, y^{(2 n-1)}(t) \leq 0$.

Dividing Eq. (2) by $y^{2 q+1}(t)$ and multiplying by $t^{2 n-1}$ and then integrating on $\left[T_{2}, t\right]$, we get

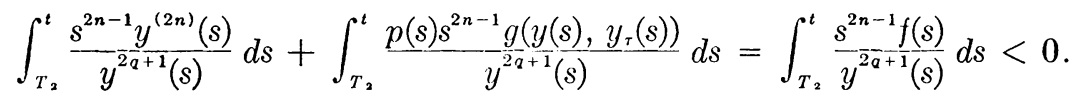

In Eq. (10) the right-hand side is negative. The second term on the left $\rightarrow \infty$ as $t \rightarrow \infty$. In fact,

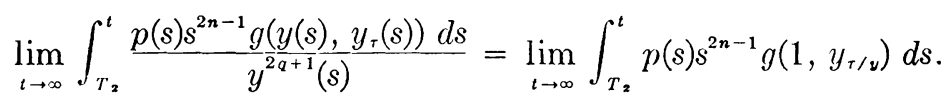

Now, by Lemma 1 applied to $-y(t)$, we find that $g(1, y \tau / y) \rightarrow g(1,1)>0$ as $t \rightarrow \infty$. Hence

$$
\lim _{t \rightarrow \infty} \int_{T_{2}}^{t} p(s) s^{2 n-1} g\left(1, \frac{y_{\tau}(s)}{y(s)}\right)=\infty .
$$

(10) and (11) imply that we must have

$$
\lim _{t \rightarrow \infty} \int_{T_{2}}^{t} \frac{s^{2 n-1} y^{(2 n)}(s)}{y^{2} \frac{z^{2+1}}{(s)}} d s=-\infty .
$$

The left-hand side of (12) is

$$
\begin{aligned}
\frac{t^{2 n-1} y^{(2 n-1)}(t)}{y^{2 q+1}(t)}-\frac{T_{2}^{2 n-1} y^{(2 n-1)}\left(T_{2}\right)}{y^{2}{ }^{2+1}\left(T_{2}\right)}-\int_{T_{2}}^{t} \frac{(2 n-1) y^{(2 n-1)}(s) s^{2 n-2}}{y^{2 q+1}(s)} d s & \\
& +\int_{T_{2}}^{t} \frac{(2 q+1) s^{2 n-1} y^{\prime}(s) y^{(2 n-1)}(s) d s}{y^{2 q+2}},
\end{aligned}
$$

in which the first and last terms are positive in view of (9a) and the second term is finite. Therefore in order for (12) to hold we must have 


$$
\lim _{t \rightarrow \infty} \int_{T_{2}}^{t} \frac{y^{(2 n-1)}(s) s^{2 n-2} d s}{y^{2 q+1}(s)}=\infty
$$

In (13), again, the left-hand side is

$$
\begin{aligned}
\frac{y^{(2 n-2)}(t) t^{2 n-2}}{y^{2 q+1}(t)}-\frac{y^{(2 n-2)}\left(T_{2}\right) T_{2}^{2 n-2}}{y^{2 q+1}\left(T_{2}\right)}-\int_{T_{2}}^{t} \frac{(2 n-2) s^{(2 n-3)} y^{(2 n-2)}(s)}{y^{2 q+1}(s)} d s & +\int_{T_{2}}^{t} \frac{s^{2 n-2} y^{(2 n-2)}(s)(2 q+1) y^{\prime}(s)}{y^{2 a+2}} d s,
\end{aligned}
$$

where the first and last terms are negative due to (9a) and the second is finite. Hence

$$
\lim _{t \rightarrow \infty} \int_{T,}^{t} \frac{s^{(2 n-3)} y^{(2 n-2)}(s) d s}{y^{2 a+1}(s)}=-\infty .
$$

Proceeding in this fashion, we find that in order for (12) to be true we must have

$$
\lim _{t \rightarrow \infty} \int_{T,}^{t} \frac{y^{\prime}(s)}{y^{2 a+1}} \frac{d s}{(s)}=\infty \text {. }
$$

But

$$
\lim _{t \rightarrow \infty} \int_{T,}^{t} \frac{y^{\prime}(s) d s}{y^{2 q+1}(s)}=\lim _{t \rightarrow \infty}\left[\frac{1}{y^{2 q}\left(T_{2}\right)}-\frac{1}{y^{2 q}(t)}\right] \frac{1}{2 q}<\infty,
$$

since $y(t)$ is finite and $q \geq 1$. This is the required contradiction and the theorem is proved.

Our next theorem generalizes Corollary 1 of Keener [4].

Theorem 2. Eq. (1) is nonoscillatory if and only if there exists a function $h(x)$ such that $h(x)<0$ and

$$
h^{(2 n)}(t)+p(t) g\left(h(t), h_{\tau}(t)\right) \geq 0
$$

for large $t$.

Proof. Suppose first that Eq. (1) is nonoscillatory. Let $y(t)$ be a nonoscillatory solution of (1). Without any loss of generality we can assume that $y(t)$ is eventually negative. Let $T_{2}$ be large enough so that for $t \geq T_{2}$, both $y(t)$ and $y_{\tau}(t)$ are negative. Since $y^{\prime}(t)<0$, there exists a number $\delta>0$ such that $y(t)+\delta<0$, and $y_{\tau}(t)+\delta<0$. Let $h(t)=y(t)+\delta<0$; then

$$
\begin{aligned}
h^{(2 n)}(t)+p(t) g\left(h(t), h_{\tau}(t)\right) & =y^{(2 n)}(t)+p(t) g\left(y(t)+\delta, y_{\tau}(t)+\delta\right) \\
& \geq y^{(2 n)}(t)+p(t) g\left(y(t), y_{\tau}(t)\right)=0 .
\end{aligned}
$$

Conversely, we take $f(t)=h^{(2 n)}(t)+p(t) g\left(h(t), h_{\tau}(t)\right)$ in Theorem 1 and arrive at $\int_{t}{ }^{\infty} t^{2 n-1} p(t) d t<\infty$ since $h<0$. This, by our next Theorem, is sufficient for Eq. (1) to be nonoscillatory.

3. Nonoscillation of Eq. (2). Theorem 3. Suppose

(i) $\quad \int^{\infty} t^{2 n-1} p(t) d t<\infty$,

(ii) $\quad \int^{\infty} t^{2 n-1} f(t) d t<\infty$;

then Eq. (2) has a bounded nonoscillatory solution.

Proof. We consider the integral equation

$$
y(t)=\frac{1}{2}+\int_{t}^{\infty} \frac{(s-t)^{2 n-1}}{(2 n-1) !} f(s) d s-\int_{t}^{\infty} \frac{(s-t)^{2 n-1}}{(2 n-1) !} p(s) g\left(y(s), y_{\tau}(s)\right) d s
$$


and set up the following estimates which are defined on $(-\infty, \infty)$ since $p$ and $f$ are defined for $x<a, a>0$.

$y_{0}(t) \equiv \frac{1}{2}$

$y_{i}(t)=\frac{1}{2}+\int_{t}^{\infty} \frac{(s-t)^{2 n-1} f(s)}{(2 n-1) !} d s-\int_{t}^{\infty} \frac{(s-t)^{2 n-1}}{(2 n-1) !} p(s) g\left(y_{i-1}(s), y_{i-1}(s-\tau(s))\right) d s$,

$$
j=1,2,3,4 \cdots .
$$

We now define sets $D, N$ in the manner of Onose [6]: $D=\left\{\left(x_{1}, x_{2}\right):\left|x_{i}\right| \leq 1, i=1,2\right\}$, $N=\{1,2, \cdots, 2 n-1\}$. Let $T$ be large enough so that for $t \geq T$

$$
\begin{gathered}
\max _{i \in N} \int_{t}^{\infty} \frac{(s-t)^{i-1}}{(i-1) !} f(s) d s \leq \frac{1}{4}, \\
\left|\left[\sup _{D} g\left(x_{1}, x_{2}\right)\right] \max _{i \in N} \int_{t}^{\infty} \frac{(s-t)^{i-1}}{(i-1) !} p(s) d s\right| \leq \frac{1}{4} .
\end{gathered}
$$

(20) and (21) are possible in view of continuity of $g$ and $f$ and the conditions of the theorem. It follows from (18), (19), (20) and (21) that

$$
\begin{aligned}
\left|y_{1}(t)\right| & \leq 1 \\
\left|y_{1}{ }^{(i)}(t)\right| & \leq \int_{t}^{\infty} \frac{(s-t)^{2 n-1-i}}{(2 n-1-i) !} f(s) d s+g\left(\frac{1}{2}, \frac{1}{2}\right) \int_{t}^{\infty} \frac{(s-t)^{2 n-1-i}}{(2 n-1-i) !} p(s) d s \\
& \leq \frac{1}{2}, \quad i=1,2,3, \cdots, 2 n-1 .
\end{aligned}
$$

In fact, it follows from (18), (19), (20), (21) and (23) that

$$
\left|y_{i}(t)\right| \leq 1,
$$

and

$$
\left|y_{i}^{(i)}(t)\right| \leq \frac{1}{2} ; j=1,2,3, \cdots ; i=1,2,3, \cdots, 2 n-1 .
$$

From (24) and (25) we find that, for $i \in\{0,1,2, \cdots, 2 n-2,2 n-1\},\left\{y_{i}^{(i)}\right\}_{i=1}{ }^{*}$ has a convergent subsequence (by Arzela's theorem) which converges uniformly to a nonoscillatory solution of Eq. (17). This is the required solution of Eq. (2). This completes the proof of this theorem.

Corollary 1. If $\int^{\infty} f(s) s^{2 n-1} d s<\infty$, then a necessary condition for Eq. (2) to oscillate is $\int^{\infty} s^{2 n-1} p(s) d s=\infty$.

Theorem 4. Suppose there exist two functions $h_{1}(t), h_{2}(t)$ such that $h_{1}(t)>0$, $h_{2}(t)>0$ eventually. Suppose further that

$$
\begin{gathered}
h_{1}{ }^{(2 n)}(t)+p(t) g\left(h_{1}(t), h_{1}(t-\tau(t)) \leq 0,\right. \\
h_{2}{ }^{(2 n)}(t)+f(t) g\left(h_{2}(t), h_{2}(t-\tau(t)) \leq 0 ;\right.
\end{gathered}
$$

then Eq. (2) is nonoscillatory.

Proof. It will be shown that $\int_{T}{ }^{\infty} t^{2 n-1} p(t) d t<\infty$ and $\int_{T}^{\infty} t^{2 n-1} f(t) d t<\infty$ for sufficiently large $T$. It is only necessary to show one of the two, say $\int_{T}{ }^{\infty} t^{2 n-1} p(t) d t<\infty$. Suppose to the contrary that $\int^{\infty} t^{2 n-1} p(t) d t=\infty$. Then let $T$ be large enough so that 
for $t \geq T, h_{1}(t)>0$ and $h_{1}(t-\tau(t))>0$. From $(26), h_{1}{ }^{(2 n)}(t) \leq 0$ for $t \leq T$. In the manner of Lemma (2), it follows that

$h_{1}(t)>0, h_{1}{ }^{\prime}(t)>0, h_{1}{ }^{\prime \prime}(t) \leq 0, h_{1}{ }^{\prime \prime \prime}(t) \geq 0, \cdots, h_{1}{ }^{(2 n-2)}(t) \leq 0, h_{1}{ }^{(2 n-1)}(t) \geq 0$.

Now in the manner of the proof of Theorem (1), it can be shown that $h_{1}(t)$ is oscillatory, a contradiction, and the proof is complete.

Theonem 5. A necessary and sufficient condition for Eq. (1) to oscillate is

$$
\int^{\infty} s^{2 n-1} p(s) d s=\infty .
$$

Corollary 2. Suppose that $\int^{\infty} s^{2 n-1} f(s) d s<\infty$. Then Eq. (1) is oscillatory if Eq. (2) is oscillatory.

Proof of Theorem 5. For the sufficiency part, suppose that (28) holds and some solution $y(t)$ of Eq. (1) is nonoscillatory. Then $y(t)$ is eventually of constant sign. Due to conditions (ii) and (iii) on the function $g$, we can assume without any loss that $y(t)$ is eventually negative. Let $T_{2}$ be so large that for $t \geq T_{2}$, both $y(t)$ and $y(t-\tau(t))$ are negative. Then it follows from Eq. (1) that for $t \geq T_{2}$

$$
y^{(2 n)}(t) \geq 0 .
$$

Therefore conclusion of Lemma (2), namely $y(t)<0, y^{\prime}(t)<0,(-1)^{i} y^{(i)}(t) \geq 0$, $i=2,3, \cdots, 2 n$, holds. Dividing Eq. (1) by $y^{2 a+1}(t)$ and multiplying by $t^{2 n-1}$ and then integrating on $\left[T_{2}, t\right]$, we get

$$
\int_{T_{2}}^{t} \frac{s^{2 n-1} y^{(2 n)}(s)}{y^{2 q+1}(s)} d s+\int_{T_{2}}^{t} \frac{p(s) s^{2 n-1} \frac{q}{2}\left(y(s), y_{\tau}(s)\right)}{y^{2-1+1}(s)} d s=0 .
$$

In Eq. (30), the second term on the left $\rightarrow \infty$ as $t \rightarrow \infty$ in the manner shown in the proof of Theorem 1. The rest of the proof is exactly identical to the remainder of the proof of Theorem 1 from Eq. (12) onward. The sufficiency is proved.

The proof of the necessity part follows in the manner of the proof of Theorem 3. Here the integral equation and the corresponding sequence of estimates are

$$
\begin{aligned}
& y(t)=1-\int_{t}^{\infty} \frac{(s-t)^{2 n-1}}{(2 n-1) !} p(s) g\left(y(s), y_{\tau}(s)\right) d s \\
& y_{0}(t) \equiv 1 \\
& y_{i}(t)=1-\int_{t}^{\infty} \frac{(s-t)^{2 n-1}}{(2 n-1) !} p(s) g\left(y_{j-1}(s), y_{j-1}(s-\tau(s)) d s, j=1,2,3, \cdots .\right.
\end{aligned}
$$

The sets $D$ and $N$ are as in Theorem 3. Let $T$ be large enough so that for $t \geq T$

$$
\left|\left[\sup _{D} g\left(x_{1}, x_{2}\right)\right] \max _{i \in N} \int_{t}^{\infty} \frac{(s-t)^{i-1}}{(i-1) !} p(s) d s\right|<\frac{1}{2} .
$$

The rest of the proof is quite similar to the proof of Theorem 3. The proof of Theorem 5 is now complete. 
Remark 1. We have, here, generalized in part the results of Gollwitzer [3] who considered the equation

$$
y^{\prime \prime}(t)+p(t) y_{\tau}{ }^{\alpha}(t)=0,
$$

where $\alpha$ is the ratio of odd integers. When $\alpha$ is an odd integer greater than 1 , a necessary and sufficient condition for (35) to oscillate is $\int^{\infty} t p(t) d t=\infty$. This follows directly from our last theorem by taking $n=1$ and $g\left(y(t), y_{\tau}(t)\right) \equiv y_{\tau}{ }^{\alpha}(t)$. The case when $\alpha=1$ was considered in the equation

$$
y^{\prime \prime}(t)+p(t) y_{\tau}(t)=0
$$

by Bradley [1] who obtained $\int^{\infty} p(t) d t=\infty$ as the sufficiency criterion for Eq. (36) oscillate. The condition obtained by us, namely $\int^{\infty} t p(t) d t=\infty$, is not sufficient for the oscillation of (36). In fact, taking $p(t)=\frac{1}{4} 1 / t^{2}$ and $\tau(t) \equiv 0$, we find that $y=\sqrt{ } t$ is a nonoscillatory solution of Eq. (36) although $\int^{\infty} t p(t) d t=\infty$.

Remark 2. All the results and their proofs remain valid in this paper if condition (ii) on $g$ is replaced by more general condition $g(\lambda x, \lambda y)=\lambda^{r} g(x, y)$, where $r>1$ and $r$ is the ratio of two odd integers. With this observation we find that Gollwitzer's [3] results for $\alpha>1$ are completely generalized.

\section{REFERENCES}

[1] John S. Bradley, Oscillation theorems for a second-order delay equation, J. Diff. Eqs. 8, 397-403 (1970)

[2] R. S. Dahiya and B. Singh, Oscillatory behavior of even-order delay equations, J. Math. Anal. Appl. 42, 183-190 (1973)

[3] H. E. Gollwitzer, On nonlinear oscillations for a second-order delay equation, J. Math. Anal. Appl. 26, 385-389 (1969)

[4] Marvin S. Keener, On the solutions of certain linear nonhomogeneous second-order differential equations, J. Applic. Anal. 1, 57-63 (1971)

[5] Michael E. Hammett, Nonoscillation properties of a nonlinear differential equation, Proc. Am. Math. Soc. 30, (1971)

[6] H. Onose, Oscillatory property of ordinary differential equations of arbitrary order, J. Diff. Eqs. 7, 454-458 (1970)

[7] B. Singh, Existence and asymptotic behavior of solutions to linear integro-differential difference equations, doctoral dissertation, University of Illinois, Urbana, 1971

[8] V. A. Staikos and A. G. Petsoulas, Some oscillation criteria for second-order nonlinear delay differential equations, J. Math. Anal. Appl. 30, 695-701 (1970) 